\title{
Instability of Hes7 protein is crucial for the somite segmentation clock
}

\author{
Hiromi Hirata $^{1,3}$, Yasumasa Bessho ${ }^{1}$, Hiroshi Kokubu ${ }^{1}$, Yoshito Masamizu${ }^{1}$, Shuichi Yamada ${ }^{1}$, Julian Lewis ${ }^{2} \&$ \\ Ryoichiro Kageyama ${ }^{1}$
}

During somitogenesis, a pair of somites buds off from the presomitic mesoderm every 2 hours in mouse embryos, suggesting that somite segmentation is controlled by a biological clock with a 2 -hour cycle ${ }^{1-3}$. Expression of the basic helix-loop-helix factor Hes7, an effector of Notch signaling, follows a 2-hour oscillatory cycle controlled by negative feedback; this is proposed to be the molecular basis for the somite segmentation clock $^{4-6}$. If the proposal is correct, this clock should depend crucially on the short lifetime of Hes7. To address the biological importance of Hes7 instability, we generated mice expressing mutant Hes7 with a longer half-life ( $\sim 30$ min compared with $\sim 22$ min for wild-type Hes7) but normal repressor activity. In these mice, somite segmentation and oscillatory expression became severely disorganized after a few normal cycles of segmentation. We simulated this effect mathematically using a direct autorepression model. Thus, instability of Hes7 is essential for sustained oscillation and for its function as a segmentation clock.

Hes7 is expressed in a cyclical fashion, and each cycle of Hes7 expression coincides with the generation of each pair of somites. Related basic helix-loop-helix genes and another Notch signaling component, lunatic fringe $(L f n g)$, show the same dynamic expression in vertebrates $^{7-18}$. We previously found that the cyclic expression of Hes7 in the presomitic mesoderm (PSM) could be mimicked by Hes1 in cultured cells ${ }^{19}$. Serum stimulation triggers an oscillation in Hes1 mRNA
Figure 1 Repressor activities and half-lives of Hes7 mutants. (a) Structure of mouse Hes7 protein. Hes7 consists of 224 amino acid residues. Seven lysine residues are indicated. (b) $\mathrm{C} 3 \mathrm{H} 10 \mathrm{~T} 1 / 2$ cells were cotransfected with the luciferase reporter gene under the control of the $\mathrm{N}$ box-containing promoter and wild-type (WT) or mutant Hes7 vectors. Relative luciferase activities are shown (mean \pm s.d. for four independent experiments). Mutants K14R, K129R and K211R had normal repressor activities, whereas the others did not.

(c) $\mathrm{C} 3 \mathrm{H} 10 \mathrm{~T} 1 / 2$ cells were transfected with the HA-tagged wild-type (WT) or mutant Hes7 expression vectors. The next day, the cells were cultured in the presence of cycloheximide for 30 min and then collected at the indicated time points. Whole-cell extracts were probed with antibody to $\mathrm{HA}$ and the relative immunoreactive activities of the signals were measured. Wild-type Hes7 is degraded with a half-life of $22.3 \mathrm{~min}$, whereas the half-lives of K14R, K129R and K211R mutant Hes7 are $30.3 \mathrm{~min}, 21.1 \mathrm{~min}$ and $15.6 \mathrm{~min}$, respectively. a

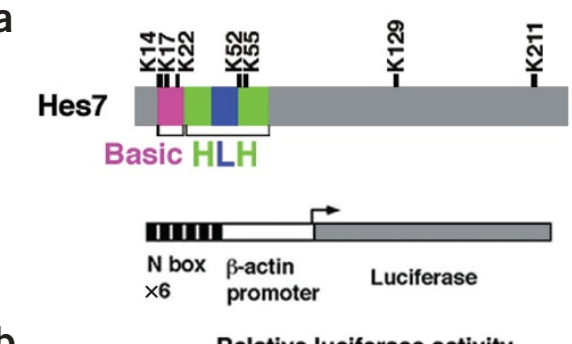

b

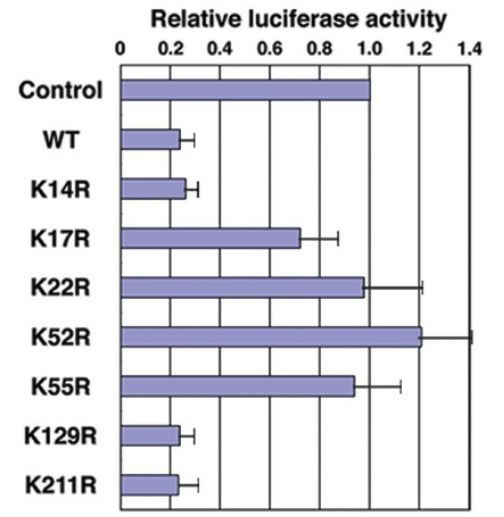

C
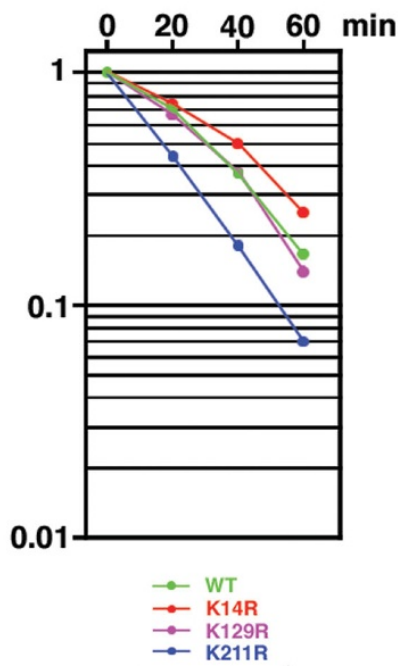

${ }^{1}$ Institute for Virus Research, Kyoto University, Shogoin-Kawahara, Sakyo-ku, Kyoto 606-8507, Japan. ${ }^{2}$ Vertebrate Development Laboratory, Cancer Research UK London Research Institute, 44 Lincoln's Inn Fields, London WC2A 3PX, UK. ${ }^{3}$ Present address: Department of Molecular, Cellular and Developmental Biology, University of Michigan, Ann Arbor 48109-1048, USA. Correspondence should be addressed to R.K. (rkageyam@virus.kyoto-u.ac.jp). 
a

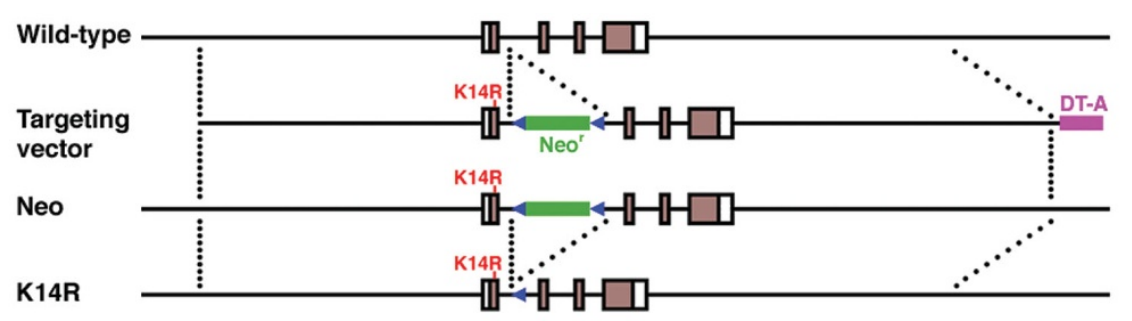

b

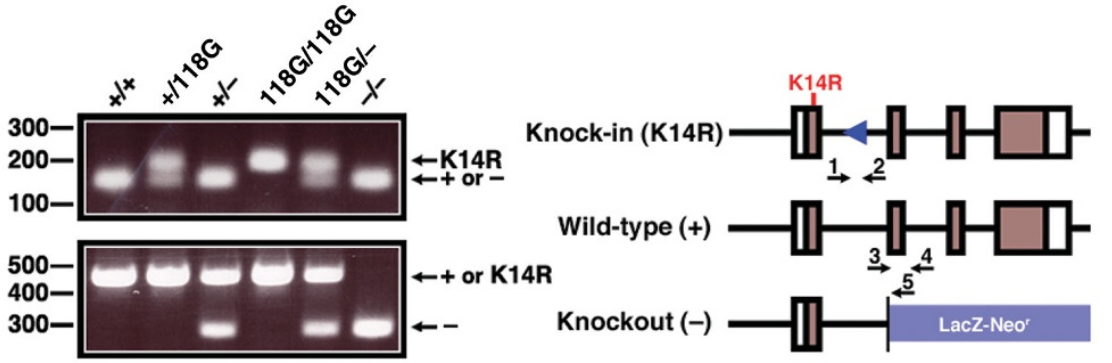

Figure 2 Generation of $H e s 7^{118 G / 118 G}$ knock-in mice for Hes7 K14R expression. (a) Targeting strategy. An $A \rightarrow G$ mutation was introduced at nucleotide residue 118 in exon 1 to code arginine instead of lysine, and the neomycin resistance gene cassette $\left(\mathrm{NeO}^{r}\right)$ was inserted into intron 1. Diphtheria toxin gene (DT-A) was used for negative selection. The blue arrowheads indicate the IoxP sites. (b) Genotype analysis by PCR. The positions of PCR primers are shown in the right panel. The upper left panel shows that PCR with primers 1 and 2 generates a 191-bp band ( Hes7118G) and a 155-bp band (wild-type or knockout). The lower left panel shows that PCR with primers 3,4 and 5 generates a 464-bp band (wild-type or Hes $7^{118 G}$ ) and a 280-bp band (knockout). and Hes1 protein levels governed by negative feedback: Hes1 protein represses Hes 1 transcription but is rapidly degraded by the proteasome system, thereby allowing the next bout of transcription. This negative feedback loop is the molecular basis for the 2-hour oscillation in Hes1 levels in cultured cells ${ }^{19}$. We recently found that the same mechanism works for Hes7 oscillation in the $\mathrm{PSM}^{5}$. The dynamic expression of Hes7 is thought to depend on its short lifespan: the half-life of Hes7 protein is only $\sim 22 \mathrm{~min}$, and this short half-life is expected to be required for genesis of the 2-hour periodicity ${ }^{5,20-22}$. To address the importance of the instability of Hes7, we manipulated its half-life.

We first screened for Hes7 mutants with long half-lives and normal repressor activity. Because lysine residues are the typical ubiquitination targets, we focused on seven lysine residues in Hes7 (Fig. 1a). We introduced a lysine-to-arginine point mutation at each lysine residue and created seven mutants (Hes7 K14R, Hes7 K17R, Hes7 K22R, Hes7 K52R, Hes7 K55R, Hes7 K129R and Hes7 K211R). To analyze the transcriptional activity of these Hes7 mutants, we carried out a transient transfection assay with the luciferase reporter gene under the control of the $\mathrm{N}$ box-containing promoter. Wild-type Hes7 repressed luciferase gene expression (Fig. 1b). Hes7 K14R, Hes7 K129R and Hes7 K211R also repressed transcription, whereas the other mutants, which have a mutation in the basic helix-loop-helix domain (Supplementary Fig. 1 online), did not repress transcription and were not studied further. We next determined the half-lives of the three Hes7 mutants with normal repressor activity. The protein half-life of wild-type Hes7 was as short as $22.3 \mathrm{~min}$, whereas those of Hes7 K14R, Hes7 K129R and Hes7 K211R were $30.3 \mathrm{~min}, 21.1 \mathrm{~min}$ and $15.6 \mathrm{~min}$, respectively (Fig. 1c). Because of its longer half-life $(30.3 \mathrm{~min})$ and normal repressor activity, we chose to study the effect of mutant Hes7 K14R on the mouse segmentation clock.

To examine the role of Hes7 half-life in somitogenesis, we generated knock-in mice expressing Hes7 K14R instead of wild-type Hes7. This was done by introducing an $\mathrm{A} \rightarrow \mathrm{G}$ point mutation at nucleotide residue 118 of Hes7 in embryonic stem (ES) cells (Fig. 2a). The

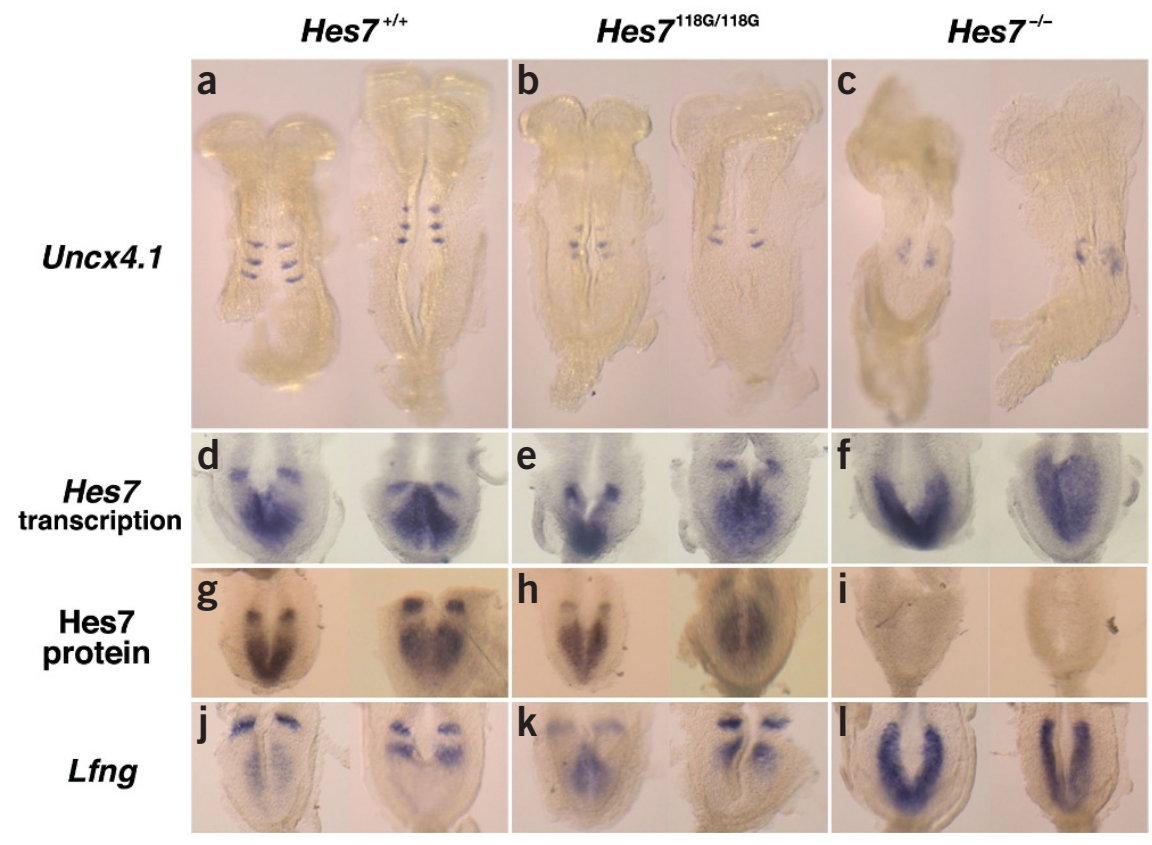

Figure 3 Dynamic expression of Hes7 K14R and Lfng at the two- or three-somite stage. (a-c) In situ hybridization with Uncx4.1 probe (marker for posterior half of each somite) indicates that the somite segmentation occurs normally in Hes $7^{118 G / 118 G}$ and $\mathrm{Hes}^{7^{+/}}$embryos but not in Hes $7^{-1-}$ embryos. (d-f) In situ hybridization with Hes7 intron probe detects the expression of nascent transcripts of all alleles $(+,-, 118 \mathrm{G})$. The expression is dynamic in $\mathrm{Hes} 7^{118 \mathrm{G} / 118 \mathrm{G}}$ and $H e s 7^{+/+}$embryos. In contrast, the nascent transcript is constitutively expressed throughout the PSM in Hes $7^{-1-}$ embryos. (g-i) Immunostaining with antibody to Hes7 shows the dynamic patterns of Hes7 protein expression in Hes $7^{118 G / 118 G}$ and Hes $7^{+/+}$embryos, whereas Hes7 protein is not expressed in Hes $7^{--}$embryos. (j-I) Lfng mRNA expression is also dynamic in $\mathrm{Hes} 7^{118 \mathrm{G} / 118 \mathrm{G}}$ and Hes $7^{+/+}$embryos but not in Hes $7^{-1-}$ embryos. Thus, the PSM in Hes $7^{118 G / 118 G}$ embryos shows periodic expression for Hes7 and Lfng oscillators. 
Figure 4 Damped oscillations of Hes7 K14R and Lfng expression at the eightsomite stage. (a-c) In situ hybridization with Uncx4.1 probe indicates that, in contrast to $\mathrm{Hes} 7^{+/+}$embryos, somite segmentation is disturbed after the threeto four-somite stage in Hes $7^{18 \mathrm{G} / 118 \mathrm{G}}$ embryos and is disturbed from the first somite stage in $\mathrm{Hes}^{7^{--}}$embryos. (d-f) Hes7 nascent transcript is constitutively expressed throughout the PSM in $\mathrm{Hes} 7^{118 \mathrm{G} / 118 \mathrm{G}}$ and $\mathrm{Hes} 7^{-/}$embryos, whereas it is expressed in a dynamic fashion in $\mathrm{Hes}^{7^{+/+}}$embryos. The expression pattern in Hes $7^{118 \mathrm{G} / 118 \mathrm{G}}$ and $\mathrm{Hes} 7^{--}$embryos is not scattered, suggesting that the oscillation is not out of synchrony but lost. (g-i) Hes7 protein is expressed in a dynamic manner in the PSM of $\mathrm{Hes}^{7^{++}}$embryos. In contrast, Hes7 protein is constitutively expressed at a low level throughout the PSM of $H e s 7^{118 G / 118 G}$ embryos and is not expressed at all in Hes $7^{-1-}$ embryos. (j-I) Lfng mRNA expression is also steady in $\mathrm{Hes} 7^{118 \mathrm{G} / 118 \mathrm{G}}$ and $\mathrm{Hes} 7^{-1-}$ embryos but periodic in $\mathrm{Hes} 7^{+/+}$embryos. Hes $7^{118 \mathrm{G} / 118 \mathrm{G}}$ and $\mathrm{Hes} \mathrm{7}^{--}$embryos show steady expression of both Hes7 intron and Lfng mRNA, but their Hes7 protein expression patterns are different.

neomycin resistance gene was excised by transient expression of Cre recombinase. From these ES cells, we generated Hes $7^{118 G / 118 G}$ knockin mice, which express Hes7 K14R (Fig. 2b). To examine cyclic gene expression in the PSM, we carried out in situ hybridization with probes for Uncx4.1, Hes7 intron and Lfng and immunostaining with antiserum to Hes7. At the two- or three-somite stage, expression of the homeobox gene Uncx4.1 showed the normal striped pattern in both Hes $7^{118 \mathrm{G} / 118 \mathrm{G}}$ and $\mathrm{Hes} 7^{+/+}$embryos but was disorganized in Hes $7^{-/-}$ embryos (Fig. 3a-c). Because intron regions are present only in nascent transcripts, in situ hybridization with intron probes identifies only the regions where the gene is actively transcribed ${ }^{5,23}$. In situ hybridization at the two- or three-somite stage with a Hes7 intron probe showed that Hes 7 was dynamically transcribed in Hes $7^{118 \mathrm{G} / 118 \mathrm{G}}$ and $H e s 7^{+/+}$embryos, whereas Hes7 transcription was constantly upregulated in $\mathrm{Hes}^{-1-}$ embryos (Fig. 3d-f). In agreement with this

\section{Phase I Phase II-III}

a

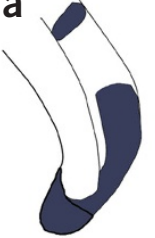

b
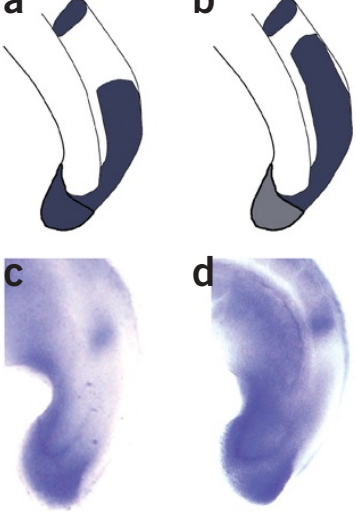

e

Hes7 $7^{186 / 1186}$

Hes7 $^{-/-}$

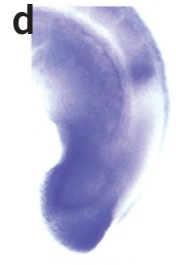

f

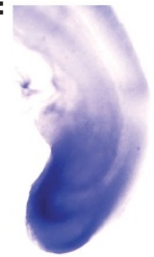

h





g

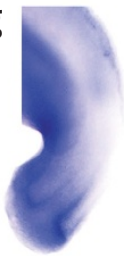

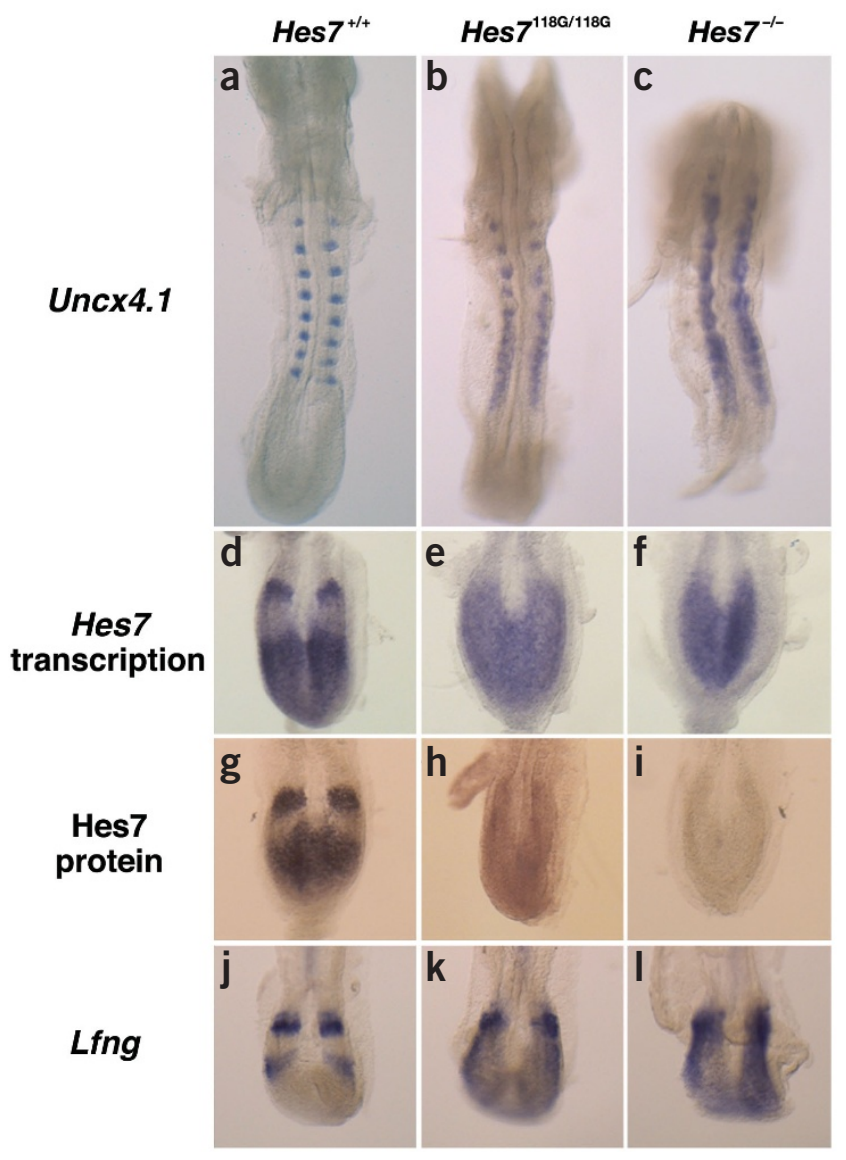

observation, we noted oscillatory expression of Hes7 protein in Hes $7^{118 \mathrm{G} / 118 \mathrm{G}}$ and Hes $7^{+/+}$embryos but not in Hes $7^{-1-}$ embryos (Fig. 3g-i). Similarly, we observed cyclic expression of $L f n g$ in $H e s 7^{118 G / 118 G}$ and $\mathrm{Hes}^{+/+}$embryos, whereas Lfng mRNA was constantly upregulated in Hes $7^{-1-}$ embryos (Fig. 3j-1). Thus, periodic gene expression in Hes $7^{118 \mathrm{G} / 118 \mathrm{G}}$ embryos occurs normally, indicating that the longer half-life of Hes7 K14R does not disrupt early somite segmentation.

To examine somite segmentation at later stages, we carried out in situ hybridization and immunostaining with embryos at the eightsomite stage. In situ hybridization with the Uncx4.1 probe showed that, in contrast to $\mathrm{Hes} 7^{+/+}$embryos, somite segmentation in Hes $7^{118 \mathrm{G} / 118 \mathrm{G}}$ embryos was disrupted after three to four somites had been generated, whereas in $H e s 7^{-1-}$ embryos, we observed fused somites throughout the entire anterior-posterior axis (Fig. 4a-c). Hes7 transcription was not periodic in either Hes $7^{118 \mathrm{G} / 118 \mathrm{G}}$ or Hes $7^{-1-}$ embryos, whereas Hes7 was transcribed dynamically in $\mathrm{Hes}^{+/+}$ embryos (Fig. $\mathbf{4 d - f}$ ). At the protein level, Hes7 expression was cyclical in $\mathrm{Hes}^{+/+}$embryos, continuous at a low level in Hes $7^{118 \mathrm{GA} 118 \mathrm{G}}$ embryos and absent in $\mathrm{Hes}^{-1-}$ embryos (Fig. 4g-i). In addition, Lfng was expressed at a steady level in both Hes $7^{118 \mathrm{G} / 118 \mathrm{G}}$ and $H e s 7^{-I-}$ embryos but was expressed periodically in $\mathrm{Hes}^{+/+}$embryos (Fig. $4 \mathbf{j}-\mathbf{l})$. In Hes $7^{118 \mathrm{G} / 118 \mathrm{G}}$ embryos, the peaks and troughs of expression became ill-defined at the four- to five-somite stage (data not shown).

Figure 5 Dynamic expression of Axin2 in Hes7 mutant mice. (a,b) Illustration of dynamic expression of Axin2 in the PSM. (c-h) In situ hybridization of Axin2 in wild-type (c,d), Hes $7^{118 G / 118 G}$ (e,f) and Hes7-null mice (g,h). Axin2 expression is still dynamic in Hes7 mutant mice (e-h), although the patterns are somewhat affected. 

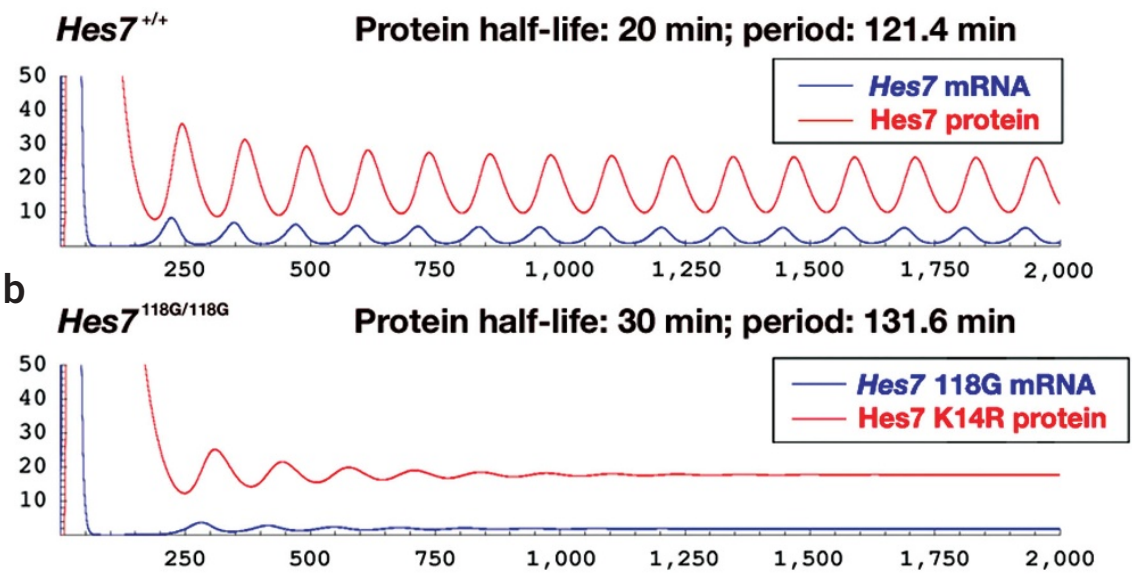

Figure 6 Mathematical simulation of Hes7 mRNA and protein oscillations. (a) For a protein half-life of $20 \mathrm{~min}$, we obtained sustained oscillations with a period of $121.4 \mathrm{~min}$. (b) For a protein halflife of $30 \mathrm{~min}$, as measured for Hes $7 \mathrm{~K} 14 \mathrm{R}$, the dynamic expression of protein and mRNA is attenuated after three to four cycles of oscillations. These values are based on data from eukaryotic cells and could be different from those of the mouse PSM. According to the mathematical theory, however, the crucial halflife, beyond which sustained oscillations become impossible (23-24 min, in this case), is largely determined simply by the period of the cycle and depends only weakly on the other parameters, whose values therefore are not essential to our conclusion. Blue and red lines indicate the relative levels of Hes7 mRNA and protein, respectively.
These results show that in Hes $7^{118 \mathrm{G} / 118 \mathrm{G}}$ embryos, periodic gene expression was maintained in early somitogenesis (one to four somites) but not at later stages. This suggests that the increase of just 8 min in Hes7 half-life disabled the oscillator, although it is also possible that the K14R mutation is hypomorphic and thereby leads to damped oscillation. Hes $7^{+/ 118 G}$ mice had a normal trunk but a kinked tail, indicating that somite segmentation was damped in these embryos, but less rapidly than in Hes $7^{118 G / 118 G}$ embryos (data not shown).

We next examined the relationship between Hes7 and Axin2, a Wntdriven oscillator ${ }^{24}$ (Fig. 5a-d). We found that Axin2 expression remained dynamic in Hes7 mutant embryos (Fig. 5e-h). On the other hand, somite segmentation seemed to occur normally in Axin2-null mice (W. Birchmeier, personal communication). These results suggest that Axin2 oscillation and Hes7 oscillation are each capable of occurring independently, but that the Hes7 oscillation is essential for segmentation. Alternatively, these oscillations could be interdependent and the damped oscillation in Hes $7^{118 \mathrm{G} / 118 \mathrm{G}}$ embryos could be due to uncoupling of Notch- and Wnt-driven oscillators.

To see whether an increase in Hes7 half-life would be expected to disrupt the segmentation clock in the way we observed, we simulated the Hes7 oscillation. The simulation is based on a recent model proposed for the zebrafish somitogenesis oscillator ${ }^{20}$. In this model, oscillations are generated by direct autorepression by its own protein product, and the transcriptional $\left(T_{\mathrm{m}}\right)$ and translational $\left(T_{\mathrm{p}}\right)$ delays (the times from initiation to completion of synthesis of an individual mRNA or protein molecule) have crucial roles. For sustained oscillation, the half-lives $\tau_{\mathrm{m}}$ and $\tau_{\mathrm{p}}$ of the mRNA and the protein, respectively, must be short compared with the sum of these delays, $T_{\mathrm{m}}+T_{\mathrm{p}}$. The period of oscillation is then $2\left(T_{\mathrm{m}}+T_{\mathrm{p}}+\left(\tau_{\mathrm{m}} / \ln 2\right)+\left(\tau_{\mathrm{p}} / \ln 2\right)\right)$, approximately $2\left(T_{\mathrm{m}}+T_{\mathrm{p}}+\right.$ $\left.1.4 \tau_{\mathrm{m}}+1.4 \tau_{\mathrm{p}}\right)$. These conditions impose quite narrow constraints on the delays and lifetimes required to give sustained oscillations with the observed 120-min period. If either of the half-lives is longer than a certain threshold value of somewhat less than $T_{\mathrm{m}}+T_{\mathrm{p}}$, sustained oscillation becomes impossible, but the system will show damped oscillations if it is started far from its steady state. Assuming that the protein halflife $\tau_{p}=20 \mathrm{~min}$, we obtained sustained oscillations with a period of $\sim 120$ min (Fig. 6a). To model the lengthening of protein half-life from $20 \mathrm{~min}$ to $30 \mathrm{~min}$, we repeated the computation substituting $\tau_{P}=30$ min but without changing other parameters. This, in essence, simulates the effect of the K14R mutation. Oscillations were no longer sustained but were damped and became severely attenuated after three to four cycles (Fig. 6b). The damped oscillations of the model thus mimic well the experimental phenomena that result from slight stabilization of Hes7 protein. Mathematical simulation with the mixed half-lives of 20 min and $30 \mathrm{~min}$ for $\mathrm{Hes}^{+/ 118 \mathrm{G}}$ also showed damped oscillation but less rapidly than for Hes $7^{118 \mathrm{G} / 118 \mathrm{G}}$, mimicking our results in heterozygous embryos (data not shown). Taken together, these simulation results and the Hes7 K14R observations suggest that the half-life of Hes7 protein is crucial for sustained oscillation as a vertebrate segmentation clock.

\section{METHODS}

Generation of Hes7 mutants. We created the Hes7 mutants by introducing point mutations in hemagglutinin (HA)-tagged Hes7 cDNA to change the lysine residue (AAA or AAG) to the arginine residue (AGA or AGG, respectively) by the Quick Change method (Stratagene). We subcloned HA-tagged wild-type or mutated Hes7 cDNAs into the eukaryotic expression vector containing the cytomegalovirus promoter, pCI-vector (Promega).

Luciferase assay. We cotransfected $\mathrm{C} 3 \mathrm{H} 10 \mathrm{~T} 1 / 2$ cells with the luciferase reporter $(0.2 \mu \mathrm{g})$ under the control of $\beta$-actin promoter with six repeats of $\mathrm{N}$ boxes with or without the expression vector for HA-tagged wild-type or mutant Hes7 $(1 \mu \mathrm{g})$ and plated them in 6-multiwell plates. We cotransfected them with the vector for Renilla luciferase under the control of the SV40 promoter $(5 \mathrm{ng}$, Promega) as an internal standard to normalize the transfection efficiency. After $24-36 \mathrm{~h}$, we collected the cells and measured luciferase activities.

Measurement of protein half-lives. We transfected C3H10T1/2 cells with expression vectors for HA-tagged wild-type or mutant Hes7. The next day, we exchanged the culture medium for one containing cycloheximide $(20 \mu \mathrm{M}$, Nacalai Tesque). Thirty minutes after the medium change (time $=0$ ) and every $20 \mathrm{~min}$ after that, we collected the cells and subjected them to western blotting with rat monoclonal antibody to HA-peroxidase (3F10, Roche; diluted 1:5,000). We visualized the immunoreactive bands with ECL Plus system (Amersham) and measured their intensities by Typhoon9410 (Amersham).

Generation of Hes $7^{118 \mathrm{G} / 118 \mathrm{G}}$ knock-in mice. All animals used in this study were maintained and handled according to protocols approved by Kyoto University. We constructed the targeting vector by replacing exon 1 with mutated exon 1 and by inserting loxP-neo-loxP into intron 1 . We identified ES cell lines with the neo allele by PCR and Southern-blot analysis. We then transiently expressed the Cre expression vector by electroporation and identified ES cell lines with the Hes $7^{118 \mathrm{G}}$ allele. We confirmed the 200-bp promoter and all the exons and introns of the Hes $7^{118 \mathrm{G}}$ allele in ES cells by sequencing. We generated chimeric mice and bred them with ICR mice. Primer sequences used for PCR are available on request. After 35 cycles of the reaction $\left(94^{\circ} \mathrm{C}\right.$ for $30 \mathrm{~s}, 68$ ${ }^{\circ} \mathrm{C}$ for $30 \mathrm{~s}$ and $72{ }^{\circ} \mathrm{C}$ for $1 \mathrm{~min}$ ), we separated the products by electrophoresis on $1.5 \%$ agarose gels. 
Whole-mount immunostaining and whole-mount in situ hybridization. We carried out whole-mount immunostaining as described previously ${ }^{25}$. We fixed embryos at embryonic day 8.0 or 8.5 with $4 \%$ paraformaldehyde in phosphatebuffered saline at $4{ }^{\circ} \mathrm{C}$ for $3 \mathrm{~h}$ and treated them with $0.1 \% \mathrm{H}_{2} \mathrm{O}_{2}$ overnight. We then incubated the embryos with antibody to Hes7 (diluted 1:100) for $5 \mathrm{~d}$ at $4^{\circ} \mathrm{C}$ and then with peroxidase-conjugated antibody to guinea pig $\operatorname{IgG}$ (Chemicon) overnight at $4{ }^{\circ} \mathrm{C}$. We visualized the peroxidase deposits with 4 chloro-1-naphthol. We carried out whole-mount in situ hybridization as described previously ${ }^{4}$. We detected Hes7 nascent transcripts with the Hes7 intron probe that corresponds to the entire first intron $(1.0 \mathrm{~kb})$.

Mathematical simulation. We computed the behavior of the Hes7 oscillator by numerically solving the following equations:

$$
\begin{aligned}
& \frac{d p(t)}{d t}=a m\left(t-T_{\mathrm{p}}\right)-b p(t) \\
& \frac{d m(t)}{d t}=f\left(p\left(t-T_{\mathrm{m}}\right)\right)-c m(t)
\end{aligned}
$$

in which $p(t)$ and $m(t)$ are the quantities of functional Hes7 protein and mRNA, respectively, per cell at time $t$, and $f(p)$ is the rate of initiation of transcription, which depends on the amount of the protein, $p$, present at the time of initiation. $a$ is the rate constant for translation, and $b$ and $c$ are the degradation rate constants for protein and $\mathrm{mRNA}$, respectively, which are simply related to the half-lives of the molecules:

$$
b=\frac{\ln 2}{\tau_{\mathrm{p}}}, \quad c=\frac{\ln 2}{\tau_{\mathrm{m}}}
$$

To represent the case where transcription is inhibited by Hes7 protein, which acts as a dimer, we assume that

$$
f(p)=\frac{k}{1+\left(\frac{p}{p_{\text {crit }}}\right)^{2}}
$$

where $k$ is the number of molecules of Hes7 mRNA synthesized per unit time in the absence of inhibition and $p_{\text {crit }}$ is the amount of protein that gives half-maximal inhibition. As in the zebrafish model ${ }^{20}$, we set $a=4.5$ protein molecules per mRNA molecule per min, $p_{\text {crit }}=40$ molecules per cell, $k=33$ mRNA molecules per cell per min, $\tau_{\mathrm{m}}=3 \mathrm{~min}$. We assume that $T_{\mathrm{m}}+T_{P}=37 \mathrm{~min}$ and that the protein half-life $\tau_{P}=20$ min for wild-type Hes7 and 30 min for Hes7 K14R.

Note: Supplementary information is available on the Nature Genetics website.

\section{ACKNOWLEDGMENTS}

We thank D. Ish-Horowicz and L. Saint-Amant for critical reading of the manuscript. This work was supported by research grants from the Ministry of Education, Culture, Sports, Science and Technology of Japan and the Japan Society for the Promotion of Science and by Cancer Research UK.

\section{COMPETING INTERESTS STATEMENT}

The authors declare that they have no competing financial interests.
Received 14 January; accepted 28 April 2004

Published online at http://www.nature.com/naturegenetics/

1. Pourquié, O. Vertebrate somitogenesis. Annu. Rev. Cell Dev. Biol. 17, 311-350 (2001)

2. Pourquié, $\mathrm{O}$. The segmentation clock: converting embryonic time into spatial pattern. Science 301, 328-330 (2003).

3. Saga, Y. \& Takeda, H. The making of the somite: molecular events in vertebrate segmentation. Nat. Rev. Genet. 2, 835-845 (2001).

4. Bessho, Y. et al. Dynamic expression and essential functions of Hes7 in somite segmentation. Genes Dev. 15, 2642-2647 (2001).

5. Bessho, Y., Hirata, H., Masamizu Y. \& Kageyama, R. Periodic repression by the bHLH factor Hes7 is an essential mechanism for the somite segmentation clock. Genes Dev. 17, 1451-1456 (2003).

6. Bessho, Y. \& Kageyama, R. Oscillations, clocks and segmentation. Curr. Opin. Genet. Dev. 13, 1678-1690 (2003).

7. Jouve, C. et al. Notch signalling is required for cyclic expression of the hairy-like gene HES1 in the presomitic mesoderm. Development 127, 1421-1429 (2000).

8. Leimeister, C. et al. Oscillating expression of c-Hey2 in the presomitic may use com binatorial signaling through multiple interacting bHLH factors. Dev. Biol. 227, 91-103 (2000).

9. Sawada, A. et al. Zebrafish Mesp family genes, mesp-a and mesp-b are segmentally expressed in the presomitic mesoderm, and Mesp-b confers the anterior identity to the developing somites. Development 127, 1691-1702 (2000).

10. Dunwoodie, S.L. et al. Axial skeletal defects caused by mutation in the spondylocostal dysplasia/pudgy gene DII3 are associated with disruption of the segmentation clock within the presomitic mesoderm. Development 129, 1795-1806 (2002).

11. Oates, A.C. \& Ho, R.K. Hairy/E(spl)-related (Her) genes are central components of the segmentation oscillator and display redundancy with the Delta/Notch signaling pathway in the formation of anterior segmental boundaries in the zebrafish. Development 129, 2929-2946 (2002).

12. Henry, C.A. et al. Two linked hairy/Enhancer of split-related zebrafish genes, her1 and her7, function together to refine alternating somite boundaries. Development 129 , 3693-3704 (2002)

13. Holley, S.A., Julich, D., Rauch, G.J., Geisler, R. \& Nüsslein-Volhard, C. her1 and the notch pathway function within the oscillator mechanism that regulates zebrafish somitogenesis. Development 129, 1175-1183 (2002).

14. McGrew, M.J., Dale, J.K., Fraboulet, S. \& Pourquié, O. The lunatic fringe gene is a target of the molecular clock linked to somite segmentation in avian embryos. Curr. Biol. 8, 979-982 (1998).

15. Forsberg, H., Crozet, F. \& Brown, N.A. Waves of mouse Lunatic fringe expression, in four-hour cycles at two-hour intervals, precede somite boundary formation. Curr. Biol. 8, 1027-1030 (1998)

16. Aulehla, A. \& Johnson, R.L. Dynamic expression of lunatic fringe suggests a link between notch signaling and an autonomous cellular oscillator driving somite segmentation. Dev. Biol. 207, 49-61 (1999).

17. Dale, J.K. et al. Periodic Notch inhibition by Lunatic Fringe underlies the chick segmentation clock. Nature 421, 275-278 (2003).

18. Serth, K., Schuster-Gossler, K., Cordes, R. \& Gossler, A. Transcriptional oscillation of Lunatic fringe is essential for somitogenesis. Genes Dev. 17, 912-925 (2003).

19. Hirata, H. et al. Oscillatory expression of the bHLH factor Hes1 regulated by a negative feedback loop. Science 298, 840-843 (2002).

20. Lewis, J. Autoinhibition with transcriptional delay: a simple mechanism for the zebrafish somitogenesis oscillator. Curr. Biol. 13, 1398-1408 (2003).

21. Monk, N.A.M. Oscillatory expression of Hes1, p53, and NF-kB driven by transcriptional time delays. Curr. Biol. 13, 1409-1413 (2003).

22. Jensen, M.H., Sneppen, K. \& Tiana, G. Sustained oscillations and time delays in gene expression of protein Hes1. FEBS Lett. 541, 176-177 (2003).

23. Morales, A.V., Yasuda, Y. \& Ish-Horowicz, D. Periodic Lunatic fringe expression is controlled during segmentation by a cyclic transcriptional enhancer responsive to notch signaling. Dev. Cel/ 3, 63-74 (2002).

24. Aulehla, A. et al. Wnt3a plays a major role in the segmentation clock controlling somitogenesis. Dev. Cell 4, 395-406 (2003).

25. Tomita, K., Moriyoshi, K., Nakanishi, S., Guillemot, F. \& Kageyama, R. Mammalian achaete-scute and atonal homologs regulate neuronal versus glial fate determination in the central nervous system. EMBO J. 19, 5460-5472 (2000). 